\title{
OBSERVACIONES SOBRE LA HISTORIA NATURAL DE CUATRO ESPECIES DE LAGARTOS \\ (SQUAMATA: DACTYLOIDAE, GEKKONIDAE, SPHAERODACTYLIDAE) EN UNA LOCALIDAD URBANA DEL OCCIDENTE DE CUBA
}

\author{
Observations on the natural history of four species of lizards \\ (Squamata: Dactyloidae, Gekkonidae, Sphaerodactylidae) in an \\ urban locality of western Cuba
}

\begin{abstract}
Luis F. de Armas
Apartado Postal 4327, San Antonio de los Baños, Artemisa 38100, Cuba. (Dorcid.org/0000-0002-9096-3382, luisdearmas1945@gmail.com
\end{abstract}

[Recibido: 11 de septiembre, 2021. Aceptado para publicación: 23 de noviembre, 2021]

\section{RESUMEN}

Se registra la muerte accidental de Anolis porcatus (Gray, 1841), Hemidactylus mabouia (Moreau de Jonnés, 1818) y Sphaerodactylus elegans (MacLeay, 1834) en el interior de vasos utilizados como adornos domésticos en un domicilio urbano de San Antonio de los Baños, provincia de Artemisa, Cuba. Además, se reportan el parasitismo del ácaro Pterygosomatidae Geckobia hemidactylii (Lawrence, 1936) en H. mabouia de esta misma localidad (primer registro para la provincia), los hábitos diurnos ocasionales de H. mabouia y S. elegans, así como la captura y muerte (no depredación) de Anolis sagrei y H. mabouia por un perro doméstico (Canis familiaris). También se presentan nuevas evidencias de coprofagia en A. porcatus y anidamiento comunal en H. mabouia.

Palabras clave: Anolis porcatus, Anolis sagrei, Hemidactylus mabouia, Sphaerodactylus elegans, conducta, reptiles sinantrópicos.

\section{ABSTRACT}

The dead of lizards belonging to three species [Anolis porcatus (Gray, 1841), Hemidactylus mabouia (Moreau de Jonnés, 1818) and Sphaerodactylus elegans (MacLeay, 1834)] into glasses used as domestic decoration in an urban residence in San Antonio de los Baños, Artemisa Province, Cuba, is recorded. Also, parasitism by the pterygosomatid mite Geckobia hemidactylii (Lawrence, 1936) on H. mabouia from this same location is reported, being this the first record for Artemisa Province. Occasional diurnal habits in H. mabouia and S. elegans are mentioned, as well as the capture and dead (not predation) of Anolis sagrei and H. mabouia by a domestic $\operatorname{dog}$ (Canis familiaris). New evidences of coprophagy in A. porcatus and communal nesting in H. mabouia are included, too.

Keywords: Anolis porcatus, Anolis sagrei, Hemidactylus mabouia, Sphaerodactylus elegans, behavior, synanthropic reptiles. 
La lista de los lagartos que en Cuba habitan regularmente en las áreas urbanas (edificios, patios, jardines, parques y áreas verdes) está integrada por tres especies de lagartijas (Anolis allisoni Barbour, 1928, A. porcatus Gray, 1837 y A. sagrei Cocteau in Duméril y Bibron, 1837), la salamanquesita Sphaerodactylus elegans (MacLeay, 1834) y los gecos Gonatodes albogularis fuscus Hallowell, 1855, Hemidactylus mabouia (Moreau de Jonnès, 1818) y H. angulatus Hallowell, 1854 (Rodríguez Schettino, 1999; Martínez Reyes y Hernández Marrero, 2003; Powell y Henderson, 2008; Borroto-Páez et al., 2015). Otras especies, mayormente introducidas, son menos abundantes o poseen una distribución mucho más limitada en el territorio nacional (Borroto-Páez et al., 2015).

En la presente contribución se presentan varias observaciones sobre la historia natural de cuatro especies de lagartos urbanos (Anolis porcatus, A. sagrei, Sphaerodactylus elegans y Hemidactylus mabouia), todas realizadas en un domicilio y su patio trasero, en el poblado de

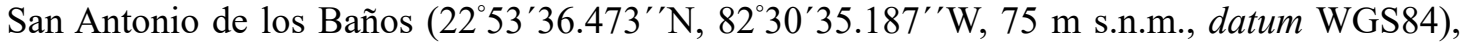
provincia de Artemisa, Cuba.

En el ambiente urbano, la muerte accidental de diversas especies de reptiles, electrocutados al ingresar en aparatos electrodomésticos, mayormente televisores y acondicionadores de aire, ha sido reportada por varios autores (Fritts y Chiszar, 1999; Chang et al., 2013; Borroto-Páez y Reyes Pérez, 2020a; Borroto-Páez et al., 2021), aunque con énfasis en las pérdidas socio-económicas que tales incidentes ocasionan, no en la conservación de las especies involucradas, algunas de ellas endémicas o nativas, aunque ninguna con categoría de amenaza.

En algunos hogares cubanos es habitual observar estantes o anaqueles en los que se exhibe una variada colección de vasos, copas y jarras, a veces con función decorativa. De forma similar a lo que sucede en ciertas luminarias, en el interior de estos recipientes de uso doméstico caen de modo casual, y mueren sin poder escapar, algunos insectos, sobre todo nocturnos, como coleópteros, lepidópteros, isópteros, ortópteros e himenópteros.

Al revisar recientemente los vasos colocados en un pequeño estante metálico, se detectaron, en el interior de tres de ellos, los cuerpos momificados de sendos reptiles juveniles (Fig. 1 a-c), pertenecientes a igual número de especies, géneros y familias: (1) Anolis porcatus (LHC: aproximadamente $19 \mathrm{~mm}$ ); (2) Hemidactylus mabouia (LHC: aproximadamente $18 \mathrm{~mm}$ ); y (3) Sphaerodactylus elegans (LHC: aproximadamente $13 \mathrm{~mm}$ ). Una posible causa de tales accidentes pudiera ser que los reptiles, atraídos por los insectos recién atrapados en esos recipientes de vidrio, intentaran capturarlos; pero una vez dentro, les fue imposible escapar.

Algunos residentes locales dijeron haber observado incidentes de esta índole, siendo H. mabouia la especie más frecuentemente involucrada en estos hechos.

Sin embargo, no es esta la única amenaza que se cierne sobre la herpetofauna urbana. Algunos animales domésticos, mayormente gatos (Rodríguez Schettino et al., 2003; Henderson y Powell, 2009; Mori et al., 2019; Borroto-Páez y Reyes Pérez, 2020b; obs. pers.) los matan y en ocasiones los depredan; peligros a los que se añade la depredación por anfibios (BorrotoPáez y Reyes Pérez, 2020b), ofidios (Armas e Iturriaga, 2017; Rodríguez-Cabrera et al., 2020), otros lagartos (Armas e Iturriaga, 2019), aves (Bello, 2000; Henderson y Powell, 2009; Guerra Solana y Armas, 2017; Armas, 2021b) y escorpiones (Armas, 2001). Y aunque más silenciosos, no pueden desestimarse la contaminación y el envenenamiento por agentes tóxicos, como los insecticidas, los cuales a veces los lagartos ingieren al comer algunas presas. 
En abril de 2021 se observó un pequeño perro (Canis familiaris) que tenía apresado entre sus fauces a un juvenil de Hemidactylus mabouia $(\mathrm{LHC}=48 \mathrm{~mm}$ ), al que dejó muerto de inmediato (Fig. 1d). Luego de su preservación en etanol $80 \%$, su examen demostró que estaba parasitado en varias partes del cuerpo (vientre, patas, cola y garganta) por numerosos ácaros Pterygosomatidae, Geckobia hemidactyli (Lawrence, 1936). Este constituye el primer registro de este ácaro para la provincia de Artemisa (véase Borroto-Páez et al., 2020).

Semanas más tarde, en horas de la tarde, se descubrió en la misma terraza el cadáver un macho subadulto de $A$. sagrei (Fig. 1f), matado por este mismo perro, presumiblemente muy temprano o el día anterior. Al amanecer del día 15 del propio mes, este perro mató otro adulto de H. mabouia $(\mathrm{LHC}=57 \mathrm{~mm})$, también parasitado por G. hemidactyli (Fig. 1e).

Aunque tanto $H$. mabouia como $S$. elegans son de hábitos eminentemente nocturnos, no es raro observarlos activos en horas del día (Martínez Reyes y Hernández Marrero, 2003). En el caso de esta última especie, durante varios años ha sido observada en actividad de caza (principalmente de moscas y hormigas) en diversas partes de la casa en horario diurno, incluso más de un individuo a la vez.

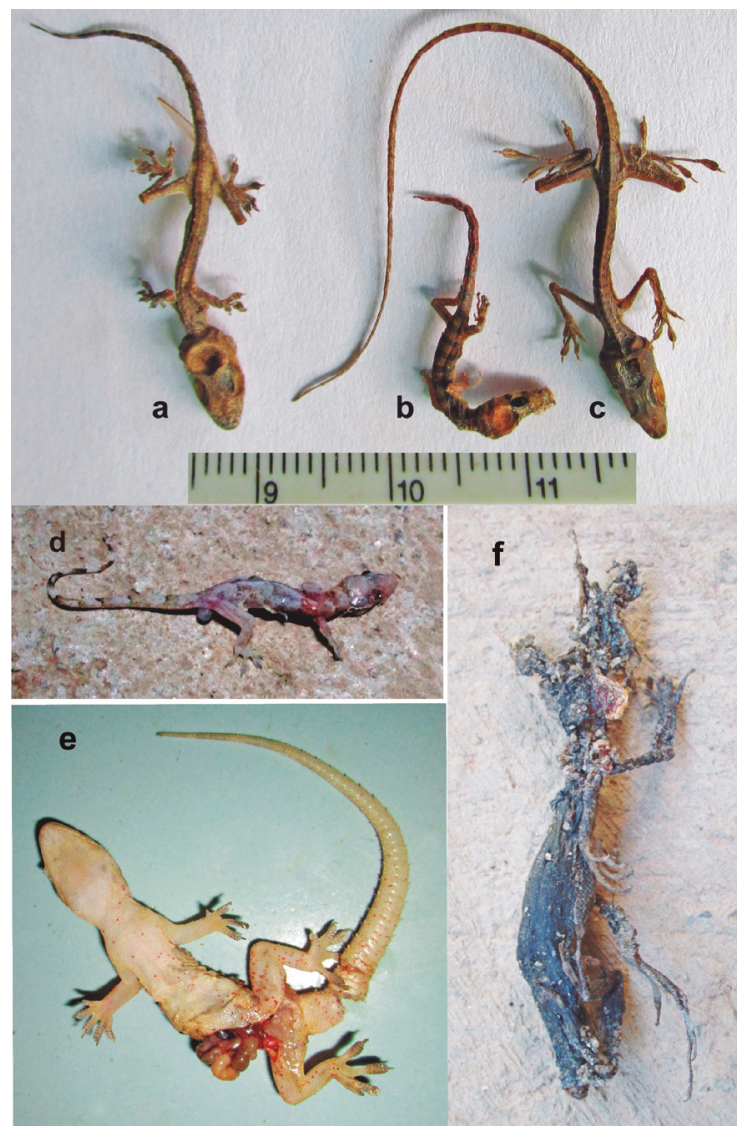

Figura 1. Lagartos muertos accidentalmente en un domicilio urbano de San Antonio de los Baños, Artemisa. a-c), cuerpos momificados hallados en el interior de sendos vasos: a, Hemidactylus mabouia; b, Sphaerodactylus elegans; c, Anolis porcatus. d-f), matados por un perro doméstico (Canis familiaris): d-e, Hemidactylus mabouia; $\mathrm{f}$, Anolis sagrei. 
Desde mediados de julio de 2020 hasta principios de mayo de 2021, fue observado un juvenil de $H$. mabouia en la cocina del hogar, el cual salía de su refugio a cualquier hora del día para cazar moscas sobre el piso (Fig. 2a). Tras su muerte, debida a un accidente doméstico, su examen permitió detectar que estaba parasitado por poco más de 100 ácaros G. hemidactyli, mayormente distribuidos en el vientre y las patas (Figs. 2 b, d). Además, su pata derecha estaba atrofiada (Fig. 2 b), a causa de un accidente relativamente reciente, pues en las fotos anteriores al mes de abril no se observa dicha deformidad. También se constató que el dedo pulgar de su mano izquierda era anómalo (Fig. 2 c). Un caso similar de hábitos diurnos fue observado durante los meses de julio y agosto de 2021; en esta oportunidad, se trataba de un juvenil, el cual pasaba gran parte del día cazando moscas sobre una mesa situada en la terraza.

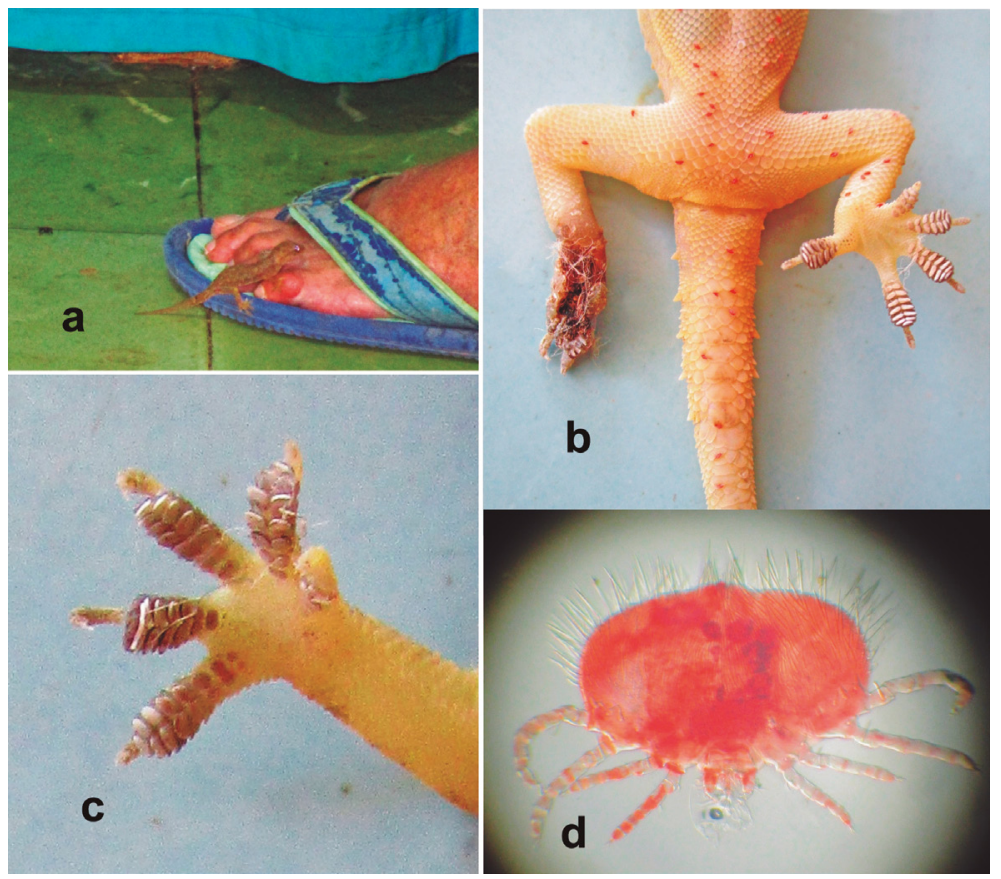

Figura 2. Hemidactylus mabouia, juvenil. a) en actividad diurna en el piso de la cocina de una vivienda urbana en San Antonio de los Baños, Artemisa. b-c) detalles del mismo espécimen después de muerto: b) patas y área pericloacal; c) mano izquierda con el dedo pulgar anómalo. d) Geckobia hemidactyli.

La coprofagia es una conducta muy pobremente documentada en los lagartos del género Anolis, siendo A. porcatus la única especie para la que se ha registrado (Armas, 2021a). El 14 de abril de 2021, a las 07:55 hr, fue observada una hembra adulta $(\mathrm{LHC}=45 \mathrm{~mm})$ de A. porcatus que, durante cerca de un minuto, estuvo lamiendo las heces frescas de un Gorrión Doméstico, Passer domesticus (Fig. 3 a). El hecho ocurrió sobre una escalera de mano, a $1.60 \mathrm{~m}$ del suelo. A las 17:20 hr del propio día, otra hembra adulta $(\mathrm{LHC}=50 \mathrm{~mm})$ estuvo lamiendo las mismas heces durante unos dos minutos (Fig. 3 b).

En conclusión, durante nueve meses, que incluyeron las estaciones de verano y la de invierno, fueron observados cinco eventos de coprofagia por A. porcatus, los cuales involucraron a dos machos y tres hembras (Armas, 2021a; este trabajo), lo cual sugiere que en esta especie dicha conducta pudiera ser relativamente frecuente. 
Las puestas comunales de H. mabouia han sido poco documentadas (Henderson y Powell, 2009). En uno de los cuartos de desahogo de la vivienda, en febrero de 2018, se detectó un anidamiento comunal de esta especie, el cual consistió en al menos 15 huevos ya eclosionados que estaban dentro de una pequeña caja de cartón de 13.0 × 13.0 x $13.5 \mathrm{~cm}$ (Fig. 3c). En septiembre de 2020, en la misma habitación, se halló otra puesta comunal, de no menos de 40 huevos (la mayoría eclosionados), depositados en el interior de una cajuela de plástico de $12.0 \times 12.0 \times 8.5 \mathrm{~cm}$ (Fig. 3d). No se tomaron las medidas de los huevos.

Entre los insectos frecuentemente reportados como presas de H. mabouia se hallan los comejenes o termitas (Iturriaga y Marrero, 2013). No obstante, los individuos sexuados (que son los únicos alados), suelen realizar sus vuelos nupciales en forma de enjambre durante unos pocos días al año (en Cuba ocurren mayormente durante los meses de primavera), por lo que constituyen una fuente de alimento irregular y fortuita para sus depredadores (Hibbitts et al., 2005).

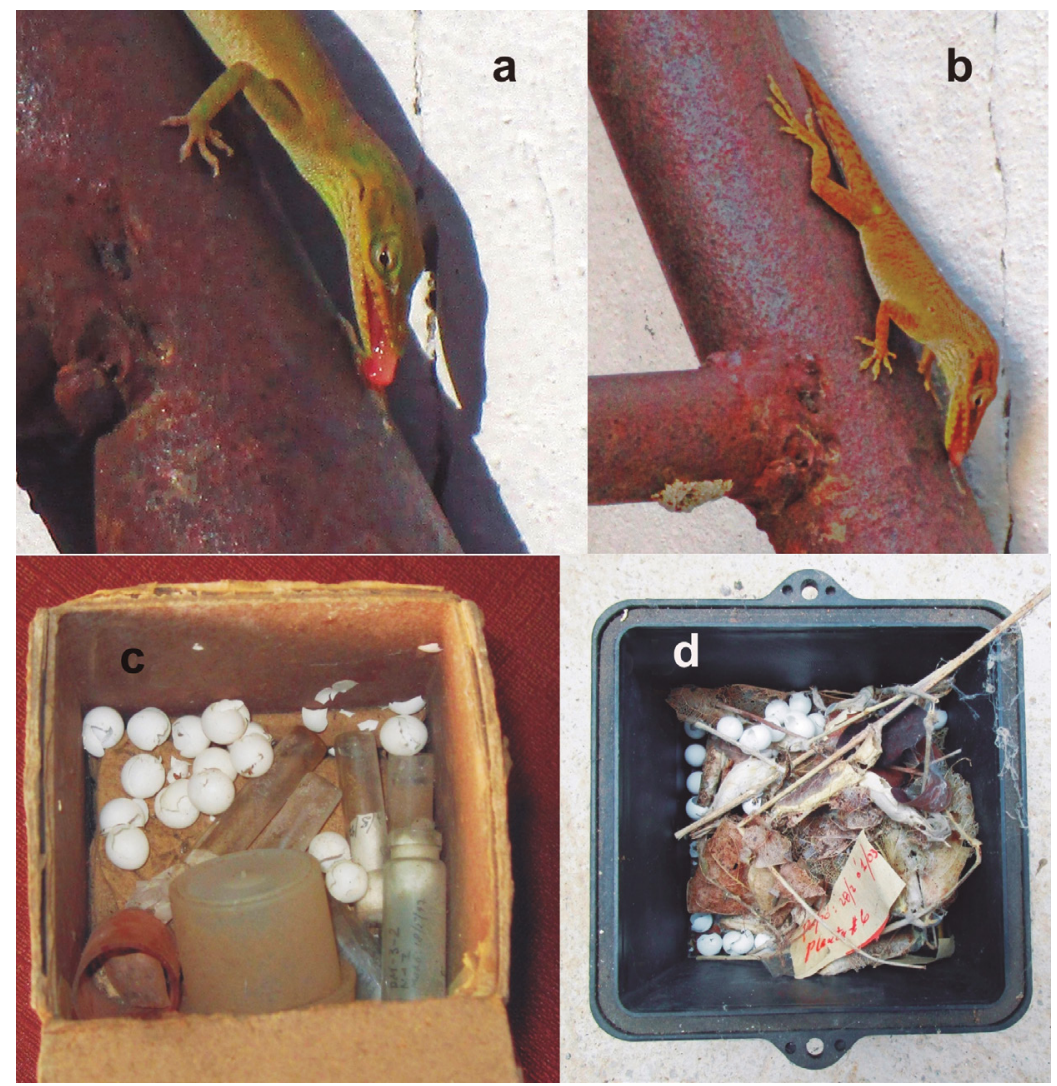

Figura 3. Coprofagia en Anolis porcatus (a-b): a, hembra lamiendo el excremento fresco de Passer domesticus; b, otra hembra, horas después, lamiendo el mismo excremento. c-d), puestas comunales de Hemidactylus mabouia en el interior de una vivienda urbana en San Antonio de los Baños, Artemisa: c, en febrero de 2018; d, en septiembre de 2020.

Durante el vuelo crepuscular de Coptotermes gestroi Wasmann, 1896 (Isoptera: Termitidae), a mediados de marzo, en una reducida área de 1.4 x $0.90 \mathrm{~m}$, fue posible observar reiteradamente hasta siete individuos de $H$. mabouia, adultos y juveniles, que ávidamente se alimentaban de estos insectos (Fig. 4 a), sin que se observara confrontación entre ellos, probablemente por la abundante disponibilidad de presas. En ocasiones, al grupo se le unió una hembra de 
A. porcatus, aunque en dos oportunidades fue atacada por los gecos. Al pie de la pared, una hembra de $A$. sagrei se alimentaba de las termitas que caían al suelo, pero nunca intentó subir a la pared, posiblemente por la fuerte competición que ello implicaría con los gecos.

El 10 de junio de 2021, a las 12:42 hr, en el fondo del patio detecté un macho de A. porcatus (LHC $\sim 60 \mathrm{~mm}$ ), que acababa de capturar una larva de Zophobas atrata (Fabricius, 1775) (Coleoptera: Tenebrionidae) de aproximadamente $28 \mathrm{~mm}$ de largo por 10-12 $\mathrm{mm}$ de ancho, la cual demoró 21 minutos en ingerir. Para lograrlo, la mantuvo agarrada por la parte anterior del cuerpo y la golpeó fuertemente, a intervalos, contra la pared, cambiando constantemente de posición, aunque preferentemente se mantuvo con la cabeza hacia abajo (Fig. 4b). Dado que estas larvas viven enterradas en un sitio donde hay materia orgánica en descomposición, es posible que la haya capturado cuando esta se expuso ligeramente en la superficie, tal vez por las altas temperaturas del suelo en esos momentos. Durante los siguientes días, después del mediodía, este lagarto perchaba más o menos en el mismo sitio, a 20-40 cm del suelo, mirando hacia donde crían estos tenebriónidos, posiblemente a la espera de que otra larva se asomara a la superficie para capturarla. Este representa el primer caso de depredación por un lagarto, en condiciones naturales, de las larvas de Z. atrata.

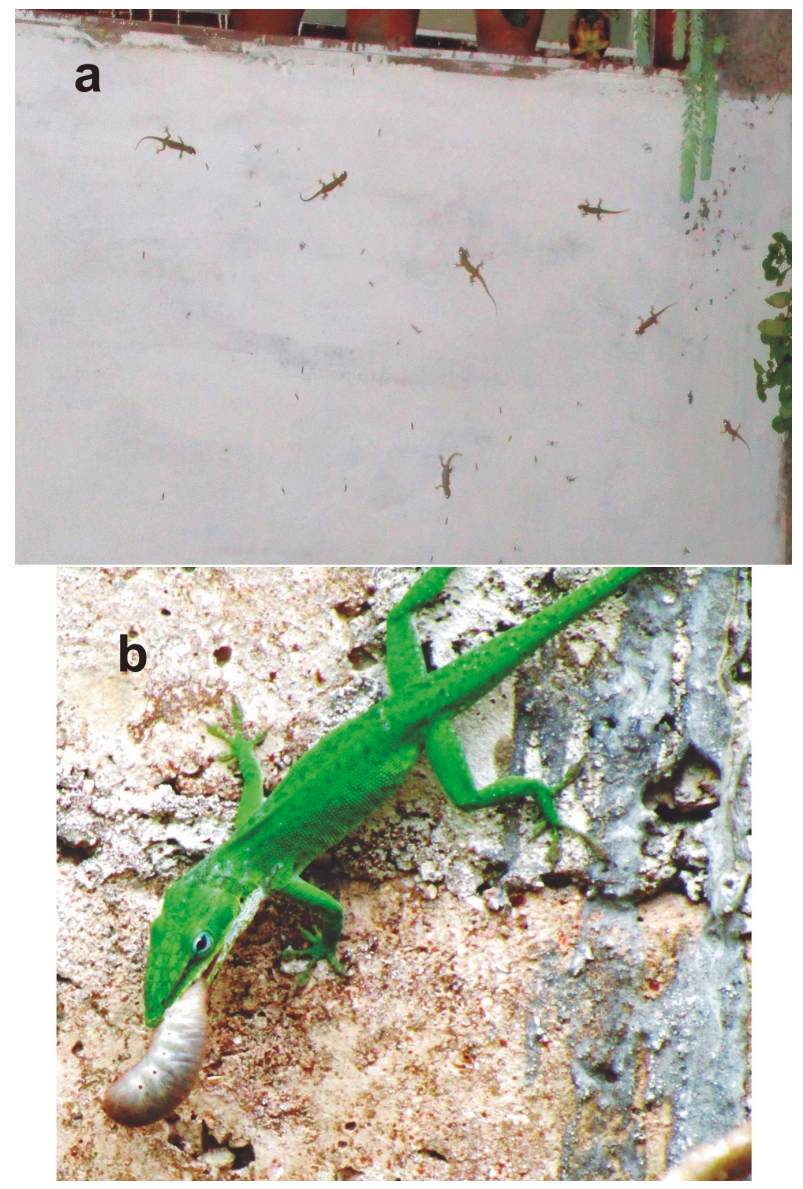

Figura 4. Depredación por Hemidactylus mabouia y Anolis porcatus. a) grupo de Hemidactylus mabouia, en marzo de 2021, a las 19:00 hr, cazando termitas aladas (Coptotermes gestroi) sobre la pared de una terraza. b) depredación de una larva de Zophobas atrata por un macho adulto de Anolis porcatus en el patio de una residencia urbana. Ambas observaciones en San Antonio de los Baños, Artemisa. 


\section{AGRADECIMIENTOS}

A Manuel Iturriaga (Instituto de Ecología y Sistemática, La Habana) por la bibliografía amablemene facilitada y la revisión crítica de la primera versión del manuscrito. A los revisores anónimos por sus oportunas y útiles sugerencias.

\section{LITERATURA CITADA}

Armas, L. F. de. 2001. Frogs and lizards as prey of some Greater Antillean arachnids. Revista Ibérica de Aracnología, 3: 87-88.

Armas, L. F. de. 2021a. Coprophagy and cannibalism in the Cuban Green Anole, Anolis porcatus Gray 1840 (Squamata: Dactyloidae). Reptiles \& Amphibians, 28 (1): 30-31.

Armas, L. F. de. 2021b. Predation of Cuban Brown Anoles, Anolis sagrei (Squamata: Dactyloidae) by House Sparrows, Passer domesticus (Aves: Passeriformes), and annotated list of lizards preyed upon by House Sparrows. IRCF Reptiles \& Amphibians, 28 (3): 432-434.

Armas, L. F. de y M. Iturriaga. 2017. Depredación de Hemidactylus mabouia (Squamata: Gekkonidae) por Tropidophis pardalis (Serpentes: Tropidophiidae). Novitates Caribaea, 11: 99-102.

Armas, L. F. de y M. Iturriaga. 2019. The Cuban Green Anole, Anolis porcatus (Squamata: Dactyloidae): catering is the best. IRCF Reptiles \& Amphibians, 26 (1): 35-38.

Bello, R. E. 2000. Anolis sp. and Gonatodes albogularis (Yellowheaded Gecko) predation. Herpetological Review, 31 (4): 239.

Borroto-Páez, R., R. Alonso Bosch, B. A. Fabres y O. Álvarez García. 2015. Introduced amphibians and reptiles in the Cuban archipelago. Herpetological Conservation and Biology, 10 (3): 985-1012.

Borroto-Páez, R., C. C. Martínez-Rivera y D. Reyes Pérez. 2020. Mites (Geckobia hemidactyli, Actinedida: Pterygosomatidae) in Tropical House Geckos (Hemidactylus mabouia) in Cuba: A review with new distribution records. IRCF Reptiles \& Amphibians, 27 (3): 382-389.

Borroto-Páez, R. y D. Reyes Pérez. 2020a. Damage to televisions and other electrical appliances by invasive and native geckos in Cuba. IRCF Reptiles \& Amphibians, 27 (1): 50-53.

Borroto-Páez, R. y D. Reyes Pérez. 2020b. Predation by a Cuban Treefrog (Osteopilus septentrionalis) and a domestic cat (Felis catus) on Tropical House Geckos (Hemidactylus mabouia) in central Cuba, with a review of predation and vertebrate prey of Tropical House Geckos. IRCF Reptiles \& Amphibians, 27 (1): 120-128.

Borroto-Páez, R., D. Reyes Pérez y B. A. Fabres. 2021. Effects of Invasive Tropical House Geckos (Hemidactylus mabouia) on Electrical Equipment: New Evidence of Damage to Televisions and Other Electrical Risks. IRCF Reptiles \& Amphibians, 28 (1): 18-21. 
Chang, C-W., J-J. Mao y G. Norval. 2013. Damage to air conditioning units caused by geckos. IRCF Reptiles \& Amphibians, 20: 143-145.

Fritts, T. y D. Chiszar. 1999. Snakes on electrical transmission lines: Patterns, causes, and strategies for reducing electrical outages due to snakes, pp. 89-103, En: Rodda, G. H., Y. Sawai, D. Chiszar y H. Tanaka (eds.), Problem Snake Management: The Habu and the Brown Treesnake. Cornell University Press, Ithaca, New York.

Guerra Solana, J. L. y L. F. de Armas. 2017. Depredación de Anolis porcatus (Squamata: Dactyloidae) por Passer domesticus (Aves: Passeriformes) en Cuba. Novitates Caribaea, 11: $103-105$.

Henderson, R.W. y R. Powell. 2009. Natural History of West Indian Reptiles and Amphibians. University Press of Florida, Gainesville, 486 pp.

Hibbitts, T. J., E. R. Pianka, R. Huey y M. J. Whiting. 2005. Ecology of the Common Barking Gecko (Ptenopus garrulus) in Southern Africa. Journal of Herpetology, 39: 509-515.

Iturriaga, M. y R. Marrero. 2013. Feeding ecology of the Tropical House Gecko Hemidactylus mabouia (Sauria: Gekkonidae) during the dry season in Havana, Cuba. Herpetology Notes, 6: 11-17.

Martínez Reyes, M. y A. Hernández Marrero. 2003. Moradores de jardines, casas y cultivos. Pp. 138-143, En Rodríguez Schettino, L. (ed.), Anfibios y reptiles de Cuba. UPC Print, Vaasa, Finlandia. 169 pp.

Mori, E., M. Menchetti, A. Camporesi, L. Cavigioli, K. Tabarelli de Fatis y M. Girardello. 2019. License to kill? Domestic cats affect a wide range of native fauna in a highly biodiverse Mediterranean country. Frontiers in Ecology and Evolution, 7: 477. https://doi.org/10.3389/fevo.2019.00477

Powell, R. y R. W. Henderson. 2008 Urban Herpetology in the West indies. pp. 87-102, En: Mitchell, J. C. y R. E. J. Brown (eds.), Urban Herpetology. Herpetological Conservation, vol. 3, Salt Lake City, Society for the Study of Amphibians and Reptiles.

Rodríguez-Cabrera, T. M., L. Y. García-Padrón, E. Morell Savall y J. Torres. 2020. Predation on direct-developing frogs (Eleutherodactylidae: Eleutherodactylus) in Cuba: New cases and a review. IRCF Reptiles \& Amphibians, 27 (2): 161-168.

Rodríguez Schettino, L. 1999. The iguanid lizards of Cuba. University Press of Florida, Miami. $428 \mathrm{pp}$.

Rodríguez Schettino, L., A. Torres Barboza y A. Hernández Marrero. 2003. Trepadores excelentes. Pp. 110-1127, En Rodríguez Schettino, L. (ed.), Anfibios y reptiles de Cuba. UPC Print, Vaasa, Finlandia, 169 pp.

Cómo citar: Armas, L. F. de. (2022). Observaciones sobre la historia natural de cuatro especies de lagartos (Squamata: Dactyloidae, Gekkonidae, Sphaerodactylidae) en una localidad urbana del Occidente de Cuba. Novitates Caribaea, (19), 126-133. https://doi.org/10.33800/nc.vi19.283. Nota científica original. 